\title{
Interface alloying effects in the magnetic properties of Fe nanoislands capped with different materials
}

\author{
Y. Huttel, ${ }^{1}$ E. Navarro, ${ }^{2}$ N. D. Telling, ${ }^{3}$ G. van der Laan,,${ }^{3,4}$ F. Pigazo, ${ }^{1}$ F. J. Palomares,${ }^{1}$ C. Quintana, ${ }^{5}$ E. Roman, ${ }^{1}$ \\ G. Armelles, ${ }^{5}$ and A. Cebollada ${ }^{5}$ \\ ${ }^{1}$ Instituto de Ciencia de Materiales de Madrid, Consejo Superior de Investigaciones Científicas (CSIC), C/ Sor Juana Inés de la Cruz, \\ 328049 Madrid, Spain \\ ${ }^{2}$ Departamento de Física de Materiales, Facultad de Físicas, Universidad Complutense, 28040 Madrid, Spain \\ ${ }^{3}$ Magnetic Spectroscopy Group, Daresbury Laboratory, Warrington WA4 4AD, United Kingdom \\ ${ }^{4}$ Diamond Light Source Ltd, Chilton, Didcot, Oxfordshire OX11 ODE, United Kingdom \\ ${ }^{5}$ Instituto de Microelectrónica de Madrid-IMM (CNM-CSIC), Isaac Newton 8 (PTM), 28760 Tres Cantos, Madrid, Spain
}

(Received 16 November 2007; revised manuscript received 11 July 2008; published 5 September 2008)

\begin{abstract}
We show that Fe nanoislands capped with $\mathrm{Al}, \mathrm{Pd}$, and Pt protecting layers include an alloy at the interface with the capping layer, which explains the previously known capping layer dependence on the interparticle magnetic coupling. Vibrating sample magnetometry results, for instance, are evidencing a reduction in the magnetization measured under a magnetic field of $15 \mathrm{mT}$, which is larger in the case of the $\mathrm{Al}$ capping and which is due to the presence of a magnetically dead interface alloy. This reduction is also observed at the atomic level using x-ray magnetic circular dichroism measurements, showing a capping layer dependence of the Fe magnetic-moment reduction that is similar for the Pd and Pt capping, and stronger for the Al capping. The trend in the magnetic properties as a function of the capping layer is explained in the light of $\mathrm{x}$-ray photoemission spectroscopy results that evidence the formation of alloys at the interface between the $\mathrm{Fe}$ nanoislands and the capping layers. The present results highlight the strong influence of interface alloying in systems of reduced dimensionality. In particular, it is shown that the magnetic properties are strongly affected at both the atomic and macroscopic level.
\end{abstract}

DOI: 10.1103/PhysRevB.78.104403

PACS number(s): 75.70. $-\mathrm{i}, 75.75 .+\mathrm{a}, 73.22 .-\mathrm{f}$

\section{INTRODUCTION}

Nanoparticles have recently attracted considerable attention due to their fascinating physicochemical properties and potential applications. For systems made of nanometric particles, the properties of the outer shell of the particles can dominate those of the core. For nanoparticles of typical size below $100 \mathrm{~nm}$, the interaction with the surrounding matrix can drastically modify the magnetic, chemical, and structural properties, converting these effects into crucial issues that have to be addressed for the implementation of nanoparticles into functional devices. Understanding the modifications of the nanoparticle outer-shell properties as a function of the surrounding matrix is therefore mandatory for their use in advanced devices.

In the specific case of magnetic nanostructures and nanometric particles, the overlayer, matrix, and outer-shell effects obviously lead to important modifications in the magnetic properties. This was first observed on metal overlayers deposited on ultrathin continuous films, e.g., the effect of overlayers of $\mathrm{Au}, \mathrm{Cu}, \mathrm{Pd}$, and $\mathrm{Ag}$ on the perpendicular magnetic anisotropy has been investigated for Co ultrathin films. ${ }^{1,2}$ Also Ag and Au overlayer thickness-driven spin reorientation transition in ultrathin and thin Co and Fe films has recently been reported. ${ }^{3-5}$ Likewise, different effects on magnetic nanoparticles have been evidenced, such as exchange bias in $\mathrm{Co}$ and $\mathrm{Fe}$ nanoparticles ${ }^{6,7}$ and tuning of the magnetic anisotropy of Co nanoparticles. ${ }^{8}$ Also the formation of alloys at the interface between nanoparticles and surrounding environment, together with their polarization and impact on the magnetic properties of the systems have been evidenced recently. ${ }^{9-11}$ The increased surface area of the nanoparticles, compared to thin films, makes the effect of the capping layer more prominent and hence more important. This makes nanoparticle assemblies ideal systems for studying the influence of the surrounding matrix and capping layer on the magnetic properties. In this paper we study the effect of the capping layer on the magnetic properties of model systems that are made of assemblies of $\mathrm{Fe}$ nanoislands deposited on $c$-sapphire substrates $\left[\mathrm{Al}_{2} \mathrm{O}_{3}(0001)\right]$ by triode sputtering. In previous in situ Kerr measurements we observed a strong effect of the Pt, Pd, and Fe capping layers on the magnetic coupling between the Fe nanoislands, but found no effect for the $\mathrm{Al}$ capping layer. ${ }^{10,11}$ These previous findings motivated further and complementary studies of the global magnetic properties of the capped nanoislands in order to elucidate the origin and the mechanism of the magnetic properties dependence on the capping layer. Vibrating sample magnetometry (VSM) measurements confirm the capping layer dependence on both the magnetic coupling and the magnetization reduction. X-ray magnetic circular dichroism (XMCD) results confirm this reduction in the $\mathrm{Fe}$ atomic magnetic moment, while X-ray photoemission spectroscopy (XPS) depth-profile analysis revealed that this is due to the formation of alloys at the interface between the Fe nanoislands and capping layers.

\section{EXPERIMENTAL DETAILS}

All samples were grown by triode sputtering in an ultrahigh-vacuum (UHV) system with base pressure in the low $10^{-9}$ mbar range. Fe nanoislands were grown while keeping the $\mathrm{Al}_{2} \mathrm{O}_{3}(0001)$ substrate at $970 \mathrm{~K}$ in order to favor 
the formation of $\mathrm{Fe}$ nanoislands, ${ }^{12,13}$ whereas the thin capping layers of $\mathrm{Fe}, \mathrm{Pt}, \mathrm{Pd}$, and $\mathrm{Al}$ (2.5 nm thick) were deposited at room temperature. The size of the nanoislands was adjusted by controlling the deposition time $\mathrm{e}^{10,11}$ and was typically $\sim 10-12 \mathrm{~nm}$ in diameter and $\sim 2.5 \mathrm{~nm}$ in height.

Transmission electron microscopy (TEM) (highresolution TEM Phillips CM200-FEG working at $200 \mathrm{keV}$ ) was used in a combined fashion to characterize the structure of the different samples. Cross-section TEM samples were prepared by the mechanical tripod polisher method ${ }^{14}$ in order to obtain large areas transparent to the electron beam. TEM measurements and selected area diffraction revealed the presence of $\mathrm{Fe}(110)$ oriented islands that presented three in-plane equivalent azimuthal orientations $\left([1 \overline{1} 0] \mathrm{Fe} \|\langle 11 \overline{2} 0\rangle \mathrm{Al}_{2} \mathrm{O}_{3}\right)$ as expected from the symmetry of the substrate. X-ray diffraction measurements revealed that the grain size was very close to the total island height as expected considering the elevated growth temperature and the small amount of Fe deposit. A more detailed structural characterization of the Fe nanoislands systems, where in particular it is shown that the Fe nanoislands have a monocrystalline bcc structure, can be found elsewhere. ${ }^{12,13}$

In situ magneto-optical transverse Kerr loops were performed on uncapped and capped $\mathrm{Fe}$ islands immediately after deposition while keeping the samples under base pressure in the low $10^{-9}$ mbar range. All Kerr measurements were performed at room temperature. The absence of in-plane magnetic anisotropy was inferred from the observation of identical hysteresis loops obtained in angle-resolved Kerr measurements.

The global magnetic properties were investigated by using VSM. All the measurements were performed by applying the magnetic field in the plane of the sample. Hysteresis loops were measured with a maximum magnetic field of 0.2 $\mathrm{T}$ from 10 to $290 \mathrm{~K}$. The temperature dependence of the magnetization was measured in zero-field- and field-cooling (ZFC/FC) measurements under a magnetic field of $150 \mathrm{Oe}$ $(15 \mathrm{mT})$. Prior to the hysteresis and ZFC/FC measurements the system was degaussed in order to eliminate remanent fields in the sample space region. Substrate diamagnetic contribution has been subtracted and data were normalized to the sample area.

$\mathrm{XMCD}$, which is a powerful technique for probing the magnetic properties of nanoparticles, was used here to obtain information about the $\mathrm{Fe}$ atomic magnetic moments. X-ray absorption spectra (XAS) were measured in total electron yield mode with $70 \%$ circularly polarized $\mathrm{x}$ rays in a reversible applied magnetic field using the Flipper chamber on station 1.1 of the Synchrotron Radiation Source (SRS) at Daresbury Laboratory, U.K. The measurements were performed at room temperature and the XMCD was obtained by subtracting XAS spectra with opposite magnetization direction.

XPS depth-profile measurements were performed in a separate UHV chamber with a base pressure of $1 \times 10^{-10}$ mbar. The excitation x-ray source was $\operatorname{Mg} K \alpha$ $(1253.6 \mathrm{eV})$ and the angle between the hemispherical analyzer (Specs-PHOIBOS100) and the plane of the surface was kept at $60^{\circ}$. XPS spectra were recorded each time after re-
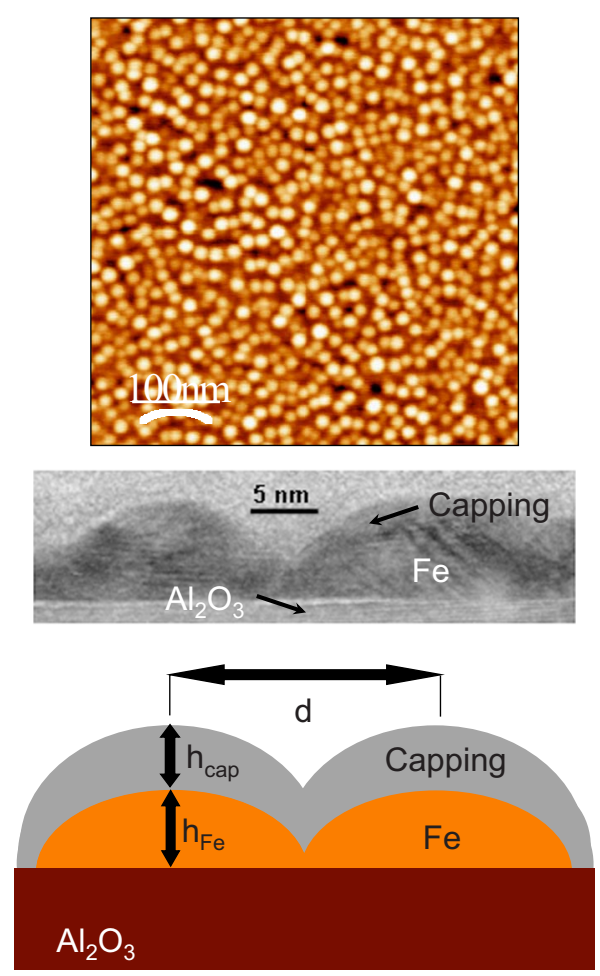

FIG. 1. (Color online) Representative AFM (top) and highresolution cross-section TEM (center) images of the Fe nanoislands covered by Pt. Corresponding model (bottom) derived from these observations.

moving very thin layers of materials from the samples by gentle $\mathrm{Ar}^{+}$sputtering at $1 \mathrm{keV}$ with calibrated ion-sputtering rate. ${ }^{15}$ This technique provides information on the chemical composition and electronic structure of the system at different depths from the surface. Prior to the data analysis, the contributions of the $\operatorname{Mg} K \alpha$ satellite lines were subtracted and the spectra were subjected to a Shirley-type background subtraction.

\section{RESULTS AND DISCUSSION}

\section{A. Transmission electron microscopy: Fe nanoislands morphology}

We have previously observed the formation of Fe nanoislands assemblies when deposited, as mentioned above, on $\mathrm{Al}_{2} \mathrm{O}_{3}(0001)$ substrates. ${ }^{12,13}$ In Fig. 1 we show representative atomic force microscopy (AFM) and high-resolution crosssection TEM images of these Fe nanoislands covered by $\mathrm{Pt}$ capping layers, together with the corresponding model of the Fe nanoisland assemblies. The AFM and TEM images illustrate the typical size, aspect ratio, distribution, and morphology observed in all samples covered by the different capping elements.

The TEM images clearly revealed the formation of $\mathrm{Fe}$ nanoislands with semispherical shape having some connection at the basis. ${ }^{10,11,13}$ From the TEM images, we defined a model for the nanoislands assemblies, which is displayed at the bottom of Fig. 1. In the present study, the geometrical parameters were as follows: $\mathrm{Fe}$ nanoislands height 


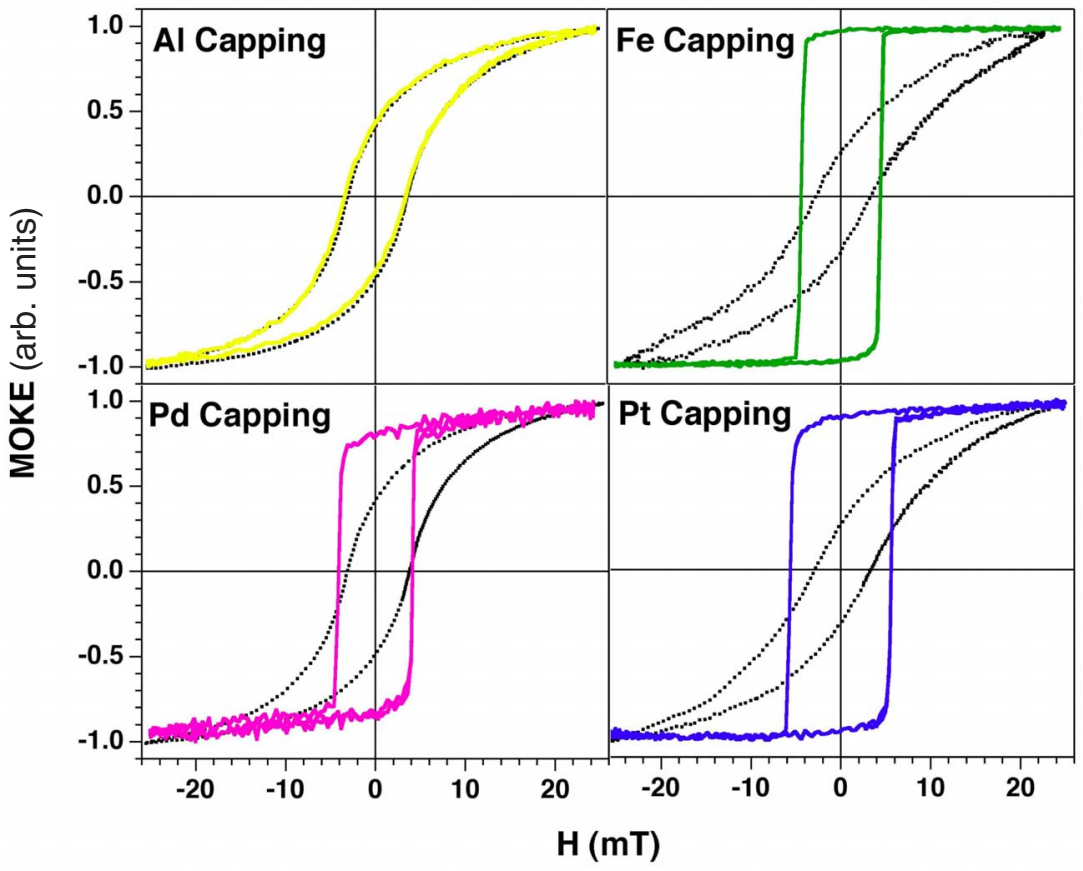

FIG. 2. (Color online) Normalized in situ transverse Kerr loops for uncapped $\mathrm{Fe}$ islands (dashed lines) with an average diameter of $12 \mathrm{~nm}$ and covered with $\mathrm{Al}, \mathrm{Fe}$, $\mathrm{Pd}$, and $\mathrm{Pt}$ capping layers grown at room temperature (continuous lines).
$h_{\mathrm{Fe}} \approx 2.5 \mathrm{~nm}$, capping layer thickness $h_{\text {cap }} \approx 2.5 \mathrm{~nm}$, and distance between first neighbor nanoislands $d \approx 12 \mathrm{~nm}$.

\section{B. In situ transverse Kerr: magnetic coupling between nanoislands}

In the system under study, the effect of depositing capping layers of variable magnetic polarizability is to modify the interparticle magnetic coupling, with an obvious strongest coupling for Fe capping layers, weaker but clearly observable for Pd and Pt capping, and absent for Al. This is shown in Fig. 2, where in situ transverse Kerr loops for Fe nanoislands before and after the deposition of different capping layers are displayed. ${ }^{10,11}$ The same rounded shape Kerr loops measured before the deposition of the capping layers reveal the presence of an assembly of weakly interacting Fe nanoislands with a given size distribution. The magnetic field at which the magnetization reversal occurs depends on the particle size. This shape of the Kerr loop is unaltered after depositing an Al capping layer, and turns into a squared hysteresis loop for a Fe capping layer, due to a strong exchange coupling between particles induced by the continuous $\mathrm{Fe}$ layer grown on top.

The effect is similar for deposition of the magnetically polarizable transition metals, $\mathrm{Pd}$ and $\mathrm{Pt}$, also leading to the formation of ferromagnetically coupled nanoislands due to the magnetic polarization of $\mathrm{Pt}$ and $\mathrm{Pd}$ at the interface between the Fe nanoislands and the capping layer. In particular, it was found that the thickness of the polarized Pd or Pt layer was $\sim 1 \mathrm{~nm},{ }^{10,11}$ also in agreement with studies performed on $\mathrm{Pt} / \mathrm{Fe}$ multilayers and Pt films deposited on Fe. ${ }^{16,17}$ These previous findings motivated the study of the global magnetic properties of the capped nanoislands, and the possible effect on the magnetization of the system.

\section{Vibrating sample magnetometry: global magnetic properties}

VSM measurements with the magnetic field applied in the sample plane showed that the magnetization values measured under a magnetic field of $15 \mathrm{mT}$ depend on the capping layer. The different values appear to be due to the magnetic polarizability. For Pt and Pd capping layers a net higher magnetization is observed as compared to the Al capping case.

Field-cooled $\left(M_{\mathrm{FC}}\right)$ and zero-field-cooled $\left(M_{\mathrm{ZFC}}\right)$ magnetization curves were recorded for $\mathrm{Pd}, \mathrm{Pt}$, and $\mathrm{Al}$ cappings and are displayed in Fig. 3. For the Pt case, the behavior was identical to the one observed for the Pd case. While $M_{\mathrm{FC}}$ increases below $60 \mathrm{~K}$, we observe a decrease in $M_{\mathrm{ZFC}}$ below $60 \mathrm{~K}$, which indicates that the magnetization becomes inhomogeneous with decreasing temperature. The behavior for the ZFC curve is characteristic for an assembly of individual magnetic domains that are frozen and form a noncollinear magnetic structure below a specific temperature $T_{f}$, which corresponds to the freezing temperature of the spins located at the grain boundaries. ${ }^{18}$ For temperatures above $T_{f}$ the boundary spins are magnetized along the direction of the adjacent grains by exchange coupling. Thus, even if the magnetization is homogeneous inside the nanoislands, the whole system is ferromagnetic. Below $T_{f}$ the boundary spins start to freeze toward the ground state, reducing the ferromagnetic exchange between grains.

Despite the difference in capping layer, and in particular the different magnetic polarizability of the $\mathrm{Pd}(\mathrm{Pt})$ and $\mathrm{Al}$, it can be observed that for both systems $T_{f}$ is similar $\left(T_{f}\right.$ $\approx 60 \mathrm{~K})$. It is expected that the size of the nanoislands would influence the value of $T_{f}$. Thus the similar $T_{f}$ values found here can be interpreted as being due to the similar average size of the pure Fe magnetic cores for both capping layers. 

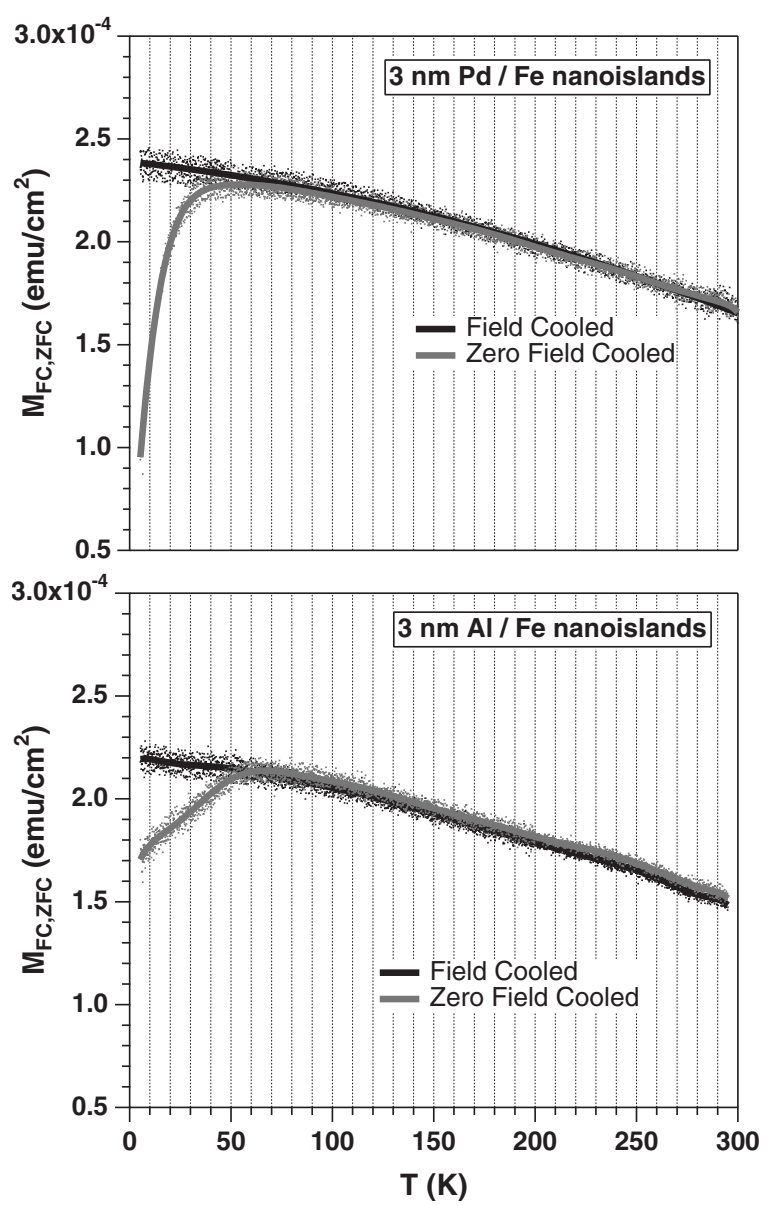

FIG. 3. Representative field-cooled and zero-field-cooled magnetization curves measured on the $\mathrm{Pd} / \mathrm{Fe}$ nanoislands/sapphire and $\mathrm{Al} / \mathrm{Fe}$ nanoislands/sapphire systems. The magnetization curves were measured with vibrating sample magnetometry.

\section{X-ray magnetic circular dichroism: interface atomic magnetic properties}

While VSM is an experimental technique that provides information on the magnetic behavior of the system as a whole, Kerr and XMCD are techniques that give more surface sensitive information. This is particularly true for XMCD due to the limited mean-free path of the electrons emitted in the $\mathrm{x}$-ray absorption process. This makes XMCD an ideal technique to study the magnetic properties of the $\mathrm{Fe}$ atoms located at the interface between the Fe nanoislands and the capping layers since the capping layer thickness was fixed at $2.5 \mathrm{~nm}$. XAS spectra for opposite magnetization directions were subtracted to obtain the XMCD spectra displayed in Fig. 4 (top panel). The qualitative differences in the $\mathrm{XMCD}$ spectra reveal the changes in the magnetic moments values.

The measured Fe $L_{2,3}$ XMCD were integrated (Fig. 4 bottom panel) in order to extract the $p$ and $q$ values, which are proportional to the atomic Fe spin $\left(\mu_{S} \propto 4 q-6 p\right)$ and orbital $\left(\mu_{L} \propto-4 q / 3\right)$ magnetic moments. ${ }^{19}$ The magnetic moments were corrected to take into account the incomplete magnetization saturation during the XMCD measurements. The results are displayed in Table I, with the values normalized to

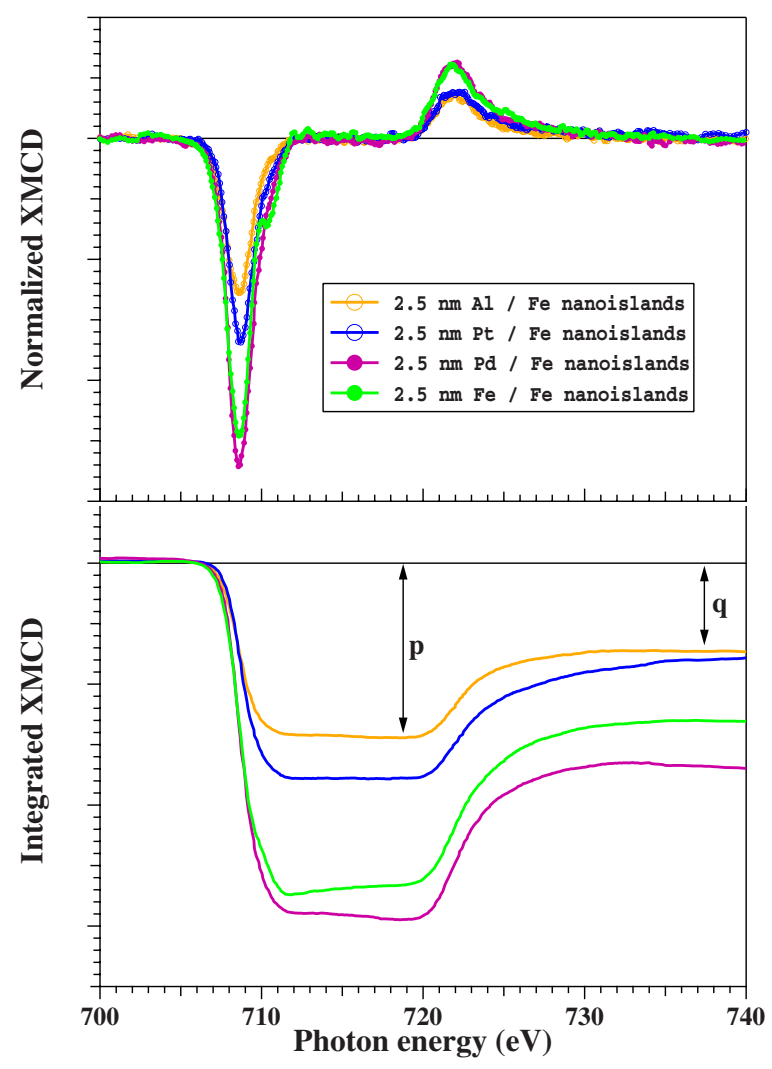

FIG. 4. (Color online) Fe $L_{2,3}$ XMCD spectra for Fe nanoisland assemblies with different capping layers (top panel). Corresponding integrated XMCD intensities (bottom panel).

the reference value for the Fe capping layer. The values given in the table for the effective spin moment $\mu_{S}$ include a contribution of the magnetic-dipole moment, $T_{z}$, as part of the sum-rule analysis. ${ }^{19}$ This contribution is usually small in bulk $3 d$ transition metals, but can become larger at surfaces and interfaces due to symmetry breaking. In principle, this contribution can be isolated using angular dependent XMCD measurements. ${ }^{20,21}$ The relative values given in Table I have been obtained assuming furthermore that the number of $d$ holes does not change significantly with the capping layer. It can be seen that in all cases there is a strong decrease in the spin and orbital magnetic moments. Surprisingly, this reduction is more important in the case of the Al capping, which apparently did not modify the magnetic properties when investigated by in situ transverse Kerr (cf., Fig. 2). The stronger reduction in the atomic magnetic moments in the case of

TABLE I. Fraction of the Fe orbital $\mu_{L}$ and spin $\mu_{S}$ magnetic moments for the nanoislands covered with different capping layers of $\mathrm{Fe}, \mathrm{Pd}, \mathrm{Pt}$, and $\mathrm{Al}$ obtained from XMCD sum-rule analysis. All values have been normalized to the case of the $\mathrm{Fe}$ capping layer.

\begin{tabular}{lcc}
\hline \hline Capping & $\mu_{L}$ & $\mu_{S}$ \\
\hline $\mathrm{Fe}$ & 1 & 1 \\
$\mathrm{Pd}$ & 0.61 & 0.35 \\
$\mathrm{Pt}$ & 0.34 & 0.33 \\
$\mathrm{Al}$ & 0.22 & 0.18 \\
\hline
\end{tabular}




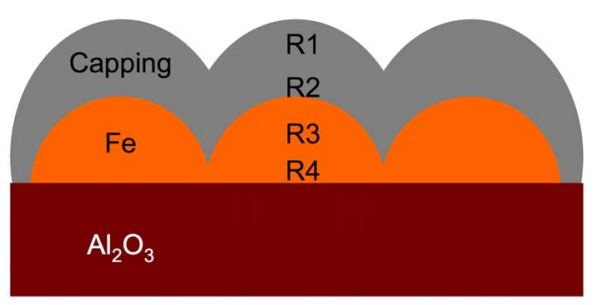

FIG. 5. (Color online) Geometrical model of the nanoislands and capping layer, together with the indication of the most relevant regions (R1, R2, R3, and R4) measured by XPS depth profiling.

the $\mathrm{Al}$ capping is in agreement with the magnetization reduction measured by VSM (see Fig. 3). The lower Fe atomic magnetic moment observed in the Pt capping case when compared to the Pd capping case is consistent with the fact that in general, the $\mathrm{Fe}$ atomic moment is lower in $\mathrm{FePt}$ alloys $^{22-24}$ than in FePd alloys. ${ }^{25,26}$ Furthermore, this is particularly true for FePt and FePd disordered alloys ${ }^{22,25,26}$ that form at low temperatures, which is likely to be the case here since the capping layers were deposited at room temperature (RT) (the ordered FePt and FePd alloys form at elevated temperatures).

The XMCD results provide direct evidence for the huge influence of the capping layer on the magnetic moments of the atoms located at the interface between the nanoislands and the capping layer. The XMCD results are consistent with the assumption that the different magnetic properties are likely to originate from the interface between the Fe nanoislands and the capping layers. However, XMCD is not per se a technique that gives layer-by-layer information and the weight of the signal arising from the outer shell of the nanoislands compared to that arising from their cores is difficult to assess.

\section{E. Depth-resolved x-ray photoemission spectroscopy: layer- resolved electronic structure}

In order to increase the understanding of the capping layer influence on the magnetic properties of the Fe nanoislands, complementary information was obtained by performing XPS depth-profile measurements. XPS spectra were recorded each time after removal of a very thin layer of material from the sample by gentle $\mathrm{Ar}^{+}$sputtering at $1 \mathrm{keV}$. This technique gives information on the electronic structure and chemical composition of the systems at different depths or different regions of the systems depicted in Fig. 5. Following this procedure, it is possible to obtain the chemical compositions of the topmost layer (region R1), at the interface between the capping layer and the nanoislands (region R2), at the center of the nanoislands (region R3), and at the interface between the nanoislands and the substrate (region R4).

Although the series of XPS spectra were measured starting from the topmost layers (region R1) down to the substrate region (region $\mathrm{R} 4$ ), for the purpose of clarity we present and discuss the XPS results starting from region R4 up to region R1. Selected Fe $2 p$ core-level XPS spectra measured at different depths are displayed in Fig. 6. While for region $\mathrm{R} 2$ we have displayed the spectra measured in each

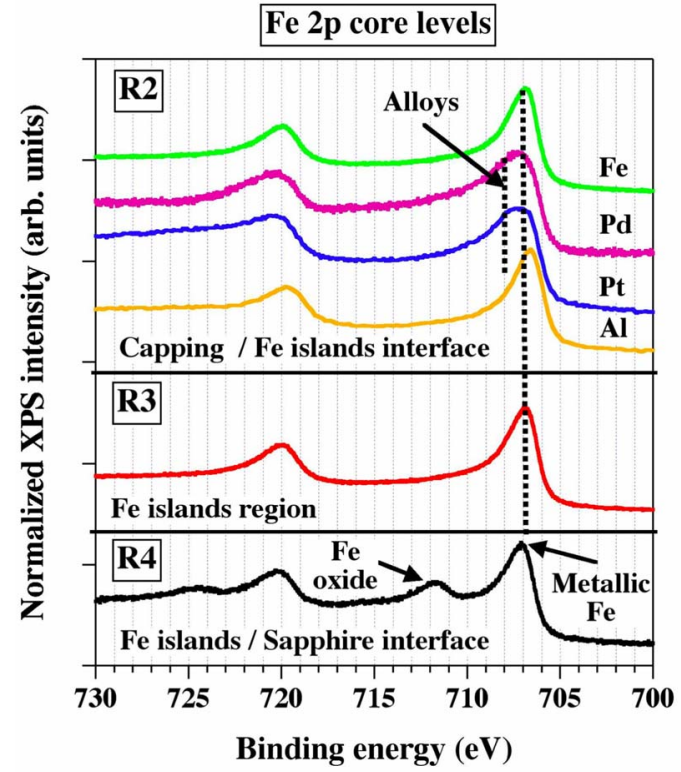

FIG. 6. (Color online) Fe $2 p$ XPS spectra of the Fe nanoisland systems on sapphire for different capping layers. The spectra were recorded in regions with different depth, i.e., distance from the surface, as indicated (R2, R3, R4, cf., Fig. 5).

sample (with capping layers of $\mathrm{Fe}, \mathrm{Pd}, \mathrm{Pt}$, and $\mathrm{Al}$ ), for regions R3 and R4 we have represented only the spectra measured in the sample with Fe capping layer since all the samples covered with the other capping elements ( $\mathrm{Pd}, \mathrm{Al}$, and $\mathrm{Pt}$ ) displayed identical spectra.

The analysis of the XPS spectra revealed that the Fe atoms in contact with the sapphire substrate are oxidized, independently of the nature of the capping layer. This can be clearly observed from the representative spectrum displayed at the bottom of Fig. 6, where the oxide contribution is marked. The oxidation of the $\mathrm{Fe}$ atoms in region $\mathrm{R} 4$ is expected due to the sample preparation method, where the iron is deposited on the sapphire substrate at $970 \mathrm{~K}$ in order to favor the formation of Fe nanoislands. While the Fe atoms in contact with the substrate exhibit an oxide component, it was observed that the center of the Fe nanoislands (region R3) is composed of pure metallic Fe, as displayed in Fig. 6, where the Fe $2 p$ spectrum exhibits only the pure metallic $\mathrm{Fe}$ component (center of Fig. 6). We now turn to the Fe $2 p$ XPS measured at the interface between the nanoislands and the capping layers (region R2). The spectra measured for the systems with different capping layers are displayed at the top of Fig. 6.

The XPS spectra recorded in region $\mathrm{R} 2$ evidenced clear changes in the electronic structure depending on the capping layer. While for the Fe capping layer there is, as expected, no change in the XPS spectrum compared to region R3 (core of the Fe nanoislands), there is a significant broadening of the core-level peaks for both Pd and Pt capping layers. Such a broadening is characteristic for the formation of $\mathrm{FePt}$ and FePd alloys. ${ }^{27,28}$ In the case of the $\mathrm{Al}$ capping, a slight corelevel shift toward lower binding energy also reveals the formation of an FeAl alloy. ${ }^{29}$ It is important to note that the presence of Fe oxides was observed only in spectra recorded 


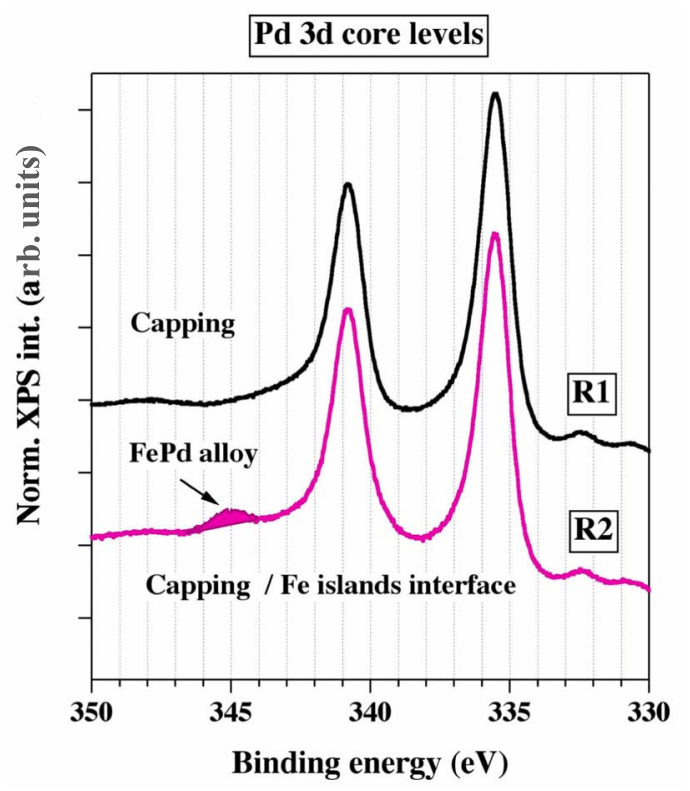

FIG. 7. (Color online) Pd $3 d$ XPS spectra for the system Pd/Fe nanoislands/sapphire recorded at different depths: Pd capping layer (R1) and interface between the Pd capping layer and the Fe nanoislands (R2).

in region $\mathrm{R} 4$ at the interface between the nanoislands and the substrate. The absence of Fe oxides in the other regions and, in particular, in region R1 clearly demonstrates the effectiveness of the capping layers against oxidation of the nanoislands.

The formation of the alloys in region R2 was also observed in the corresponding $\mathrm{Pd} 3 d$ and $\mathrm{Al} 2 p$ and $2 s$ corelevel spectra measured in the same region. Figure 7 displays the $\mathrm{Pd} 3 d$ spectrum measured in region $\mathrm{R} 2$, which is compared to the pure $\mathrm{Pd}$ reference spectrum (region R1). The presence of an additional spectral feature at a binding energy of $345 \mathrm{eV}$ is observed in region $\mathrm{R} 2$, which is absent for region $\mathrm{R} 1$. This additional feature is characteristic for the presence of a FePd alloy, ${ }^{28}$ and in agreement with Fe $2 p$ XPS results displayed in Fig. 6.

In the case of the Pt capping layer, no additional feature was observed in the $\mathrm{Pt} 4 f$ spectrum that could be assigned to the formation of a FePt alloy due to the limited experimental resolution. For the Al capping case, the formation of the $\mathrm{FeAl}$ alloy could be detected in the $\mathrm{Al} 2 s$ spectrum, which is displayed in Fig. 8. For comparison also the corresponding spectrum for region $\mathrm{R} 1$ (Al reference spectrum) is displayed.

The Al reference spectrum, that has been recorded after removing the first native $\mathrm{Al}$ oxide layers, clearly displays the characteristic Al bulk and surface-plasmon structures. ${ }^{30,31}$ As it can be observed in Fig. 8, the plasmon structures are replaced by a broader band in the spectrum for region R2, which is known to be characteristic for the formation of an FeAl alloy. ${ }^{31}$

The XPS results therefore show that the $\mathrm{Pd}, \mathrm{Pt}$, and $\mathrm{Al}$ are forming an alloy located at the interface between the $\mathrm{Fe}$ nanoislands and the capping layers. On the other hand, it is well established that Pd and Pt are magnetically polarized in contact with $\mathrm{Fe},{ }^{11,32-34}$ while the $\mathrm{Al}$ has no magnetic moment in contact with $\mathrm{Fe}^{35}$

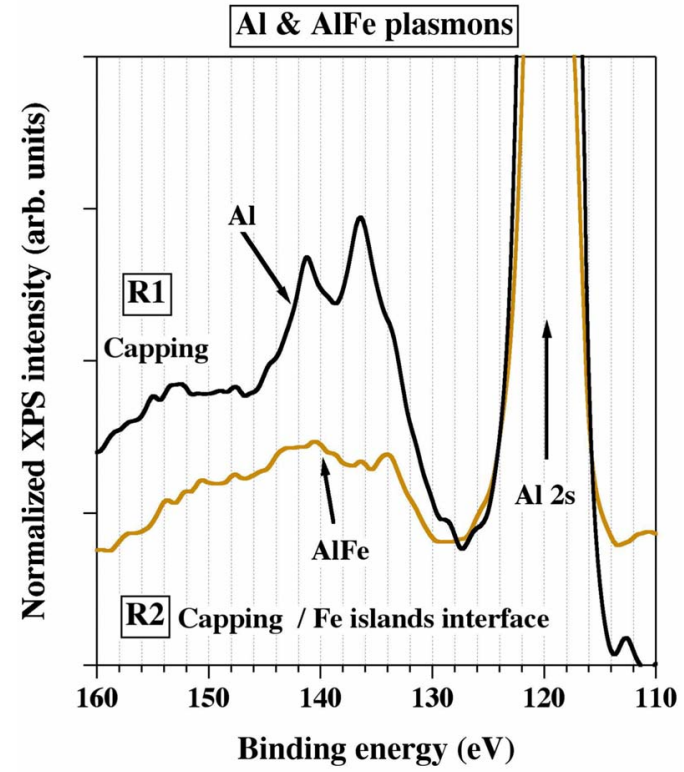

FIG. 8. (Color online) Al $2 s$ XPS spectra for the system $\mathrm{Al} / \mathrm{Fe}$ nanoislands/sapphire recorded at different depths: Al capping layer (R1) and interface between the Al capping layer and the Fe nanoislands (R2).

The alloy formation at the interface between the $\mathrm{Fe}$ nanoislands and the capping layers and the corresponding different magnetic properties of the alloys depending on the capping material explain the changes of the magnetic properties observed by Kerr, VSM, and XMCD. Both types of capping materials (polarizable $\mathrm{Pt}$ and $\mathrm{Pd}$, and nonpolarizable $\mathrm{Al}$ ) are forming alloys at the interface between the Fe nanoislands and the capping layers that in turn reduce the size of the $\mathrm{Fe}$ nanoisland cores and the effective magnetic moments of the Fe atoms as observed by XMCD. Such a reduction in the effective magnetic moments of the Fe atoms is less pronounced for the polarizable capping materials, where the Fe atoms in the formed alloy are still in a ferromagnetic configuration. The XMCD results are supported by the VSM results, where also a reduction in the effective magnetic volume in the $\mathrm{Al} / \mathrm{Fe}$ system has been observed that is more pronounced than for the $\mathrm{Pt} / \mathrm{Fe}$ and $\mathrm{Pd} / \mathrm{Fe}$ systems. Finally, the magnetic connection (for Pd or Pt capping layers) of the $\mathrm{Fe}$ nanoislands and the absence of a magnetic connection (for Al capping layer) is clearly observed with Kerr, while not highlighted by VSM due to the difference in surface sensitivity of the two techniques.

\section{CONCLUSIONS}

The origin of the effect of different capping layers ( $\mathrm{Pt}, \mathrm{Pd}$, and $\mathrm{Al}$ ) on the magnetic connection of an assembly of $\mathrm{Fe}$ nanoislands has been studied using several complementary experimental techniques. VSM has evidenced a reduction in the magnetization measured under a magnetic field of $15 \mathrm{mT}$ that is similar for $\mathrm{Pt}$ and $\mathrm{Pd}$ capping layers and in turn more 
important in the case of an Al capping layer. Such a reduction in the magnetization is confirmed at the atomic level by $\mathrm{XMCD}$ results that show a reduction in the Fe atomic magnetic moments, which is largest for the Al case. The trends of the magnetic properties have been interpreted in the light of XPS results that revealed the formation of alloys at the interfaces between the capping layers of $\mathrm{Pt}, \mathrm{Pd}$, and $\mathrm{Al}$ with the Fe nanoislands. The results presented here clearly demonstrate that alloying processes in systems with reduced dimensions are likely to be mandatory for their magnetic properties.

\section{ACKNOWLEDGMENTS}

Support from the SRS staff during the XMCD experiments is greatly acknowledged. Financial support from the Spanish Ministerio de Ciencia y Tecnología, under Contract No. MAT2005-05524-C02 is acknowledged. Y. H. also acknowledges financial support from the "Ramón y Cajal" program, Consejo Superior de Investigaciones Científicas (CSIC) and Comunidad Autónoma de Madrid (CAM) under Project No. S-0505/MAT/0194 NANOMAGNET.
${ }^{1}$ B. N. Engel, M. H. Wiedmann, and C. M. Falco, J. Appl. Phys. 75, 6401 (1994).

${ }^{2}$ P. Beauvillain, A. Bounouh, C. Chappert, R. Mégy, S. OuldMahfoud, J. P. Renard, and P. Veillet, J. Appl. Phys. 76, 6078 (1994).

${ }^{3}$ M. Kisielewski, A. Maziewski, M. Tekielak, A. Wawro, and L. T. Baczewski, Phys. Rev. Lett. 89, 087203 (2002).

${ }^{4}$ I.-G. Baek, H. G. Lee, H. J. Kim, and E. Vescovo, Phys. Rev. B 67, 075401 (2003).

${ }^{5}$ F. Zavaliche, W. Wulfhekel, M. Przybylski, S. Bodea, J. Grabowski, and J. Kirschner, J. Phys. D 36, 779 (2003).

${ }^{6}$ V. Skumryev, S. Stoyanov, Y. Zhang, G. Hadjipanayis, D. Givord, and J. Nogués, Nature (London) 423, 850 (2003).

${ }^{7}$ L. Del Bianco, D. Fiorani, A. M. Testa, E. Bonetti, and L. Signorini, Phys. Rev. B 70, 052401 (2004).

${ }^{8}$ F. Luis, F. Bartolomé, F. Petroff, J. Bartolomé, L. M. García, C. Deranlot, H. Jaffrès, M. J. Martínez, P. Bencok, F. Wilhelm, A. Rogalev, and N. B. Brookes, Europhys. Lett. 76, 142 (2006).

${ }^{9}$ J. Bartolomé, L. M. García, F. Bartolomé, F. Luis, F. Petroff, C. Deranlot, F. Wilhelm, and A. Rogalev, J. Magn. Magn. Mater. 316, e9 (2007).

${ }^{10}$ E. Navarro, Y. Huttel, C. Clavero, G. Armelles, and A. Cebollada, Appl. Phys. Lett. 84, 2139 (2004).

${ }^{11}$ E. Navarro, Y. Huttel, C. Clavero, A. Cebollada, and G. Armelles, Phys. Rev. B 69, 224419 (2004).

${ }^{12}$ J. L. Menéndez, G. Armelles, C. Quintana, and A. Cebollada, Surf. Sci. 482-485, 1135 (2001).

${ }^{13}$ C. Quintana, J. L. Menéndez, Y. Huttel, M. Lancin, E. Navarro, and A. Cebollada, Thin Solid Films 434, 228 (2003).

${ }^{14} \mathrm{~J}$. Benedict, R. Anderson, and S. J. Klepeis, in Specimen Preparation for Transmission Electron Microscopy of Materials II, MRS Symposia Proceedings No. 199, edited by R. Anderson (Materials Research Society, Pittsburgh, 1990), p. 189.

${ }^{15}$ M. S. Martín-González, Y. Huttel, A. Cebollada, G. Armelles, and F. Briones, Surf. Sci. 571, 63 (2004).

${ }^{16}$ W. J. Antel, M. M. Schwickert, T. Lin, W. L. OBrien, and G. R. Harp, Phys. Rev. B 60, 12933 (1999).

${ }^{17}$ R. Bertacco and F. Ciccacci, Phys. Rev. B 57, 96 (1998).

${ }^{18}$ Z. Sefrioui, J. L. Menéndez, E. Navarro, A. Cebollada, F. Briones, P. Crespo, and A. Hernando, Phys. Rev. B 64, 224431 (2001).

${ }^{19}$ B. T. Thole, P. Carra, F. Sette, and G. van der Laan, Phys. Rev. Lett. 68, 1943 (1992); P. Carra, B. T. Thole, M. Altarelli, and X.
Wang, ibid. 70, 694 (1993); C. T. Chen, Y. U. Idzerda, H.-J. Lin, N. V. Smith, G. Meigs, E. Chaban, G. H. Ho, E. Pellegrin, and F. Sette, ibid. 75, 152 (1995).

${ }^{20}$ J. Stöhr and H. König, Phys. Rev. Lett. 75, 3748 (1995).

${ }^{21}$ H. A. Dürr and G. van der Laan, Phys. Rev. B 54, R760 (1996).

${ }^{22}$ E. E. Alp, M. Ramanathan, S. Salem-Sugui, Jr., F. Oliver, V. Stojanoff, and D. P. Siddons, Rev. Sci. Instrum. 63, 1221 (1992).

${ }^{23}$ C. Antoniak, J. Lindner, M. Spasova, D. Sudfeld, M. Acet, M. Farle, K. Fauth, U. Wiedwald, H. G. Boyen, P. Ziemann, F. Wilhelm, A. Rogalev, and S. Sun, Phys. Rev. Lett. 97, 117201 (2006).

${ }^{24}$ N. Jaouen, D. Babonneau, J. M. Tonnerre, D. Carbone, F. Wilhelm, A. Rogalev, T. K. Johal, and G. van der Laan, Phys. Rev. B 76, 104421 (2007).

${ }^{25}$ I. Galanakis, S. Ostanin, M. Alouani, H. Dreyssé, and H. Ebert, Comput. Mater. Sci. 17, 455 (2000).

${ }^{26}$ P. Kamp, A. Marty, B. Gilles, R. Hoffmann, S. Marchesini, M. Belakhovsky, C. Boeglin, H. A. Dürr, S. S. Dhesi, G. van der Laan, and A. Rogalev, Phys. Rev. B 59, 1105 (1999).

${ }^{27}$ H.-G. Boyen et al., Adv. Mater. (Weinheim, Ger.) 17, 574 (2005).

${ }^{28}$ J.-H. Choi, T.-U. Nahm, Wookje Kim, Wondong Kim, J. Chung, J.-Y. Kim, H. Koh, and S.-J. Oh, Surf. Sci. 495, 173 (2001).

${ }^{29}$ H. Graupner, L. Hammer, K. Heinz, and D. M. Zehner, Surf. Sci. 380, 335 (1997).

${ }^{30}$ L. P. H. Jeurgens, W. G. Sloof, C. G. Borsboom, F. D. Tichelaar, and E. J. Mittemeijer, Appl. Surf. Sci. 161, 139 (2000).

${ }^{31}$ M. Oku, N. Masahashi, S. Hanada, and K. Wagatsuma, J. Alloys Compd. 413, 239 (2006).

${ }^{32}$ J. Vogel, A. Fontaine, V. Cros, F. Petroff, J. P. Kappler, G. Krill, A. Rogalev, and J. Goulon, J. Magn. Magn. Mater. 165, 96 (1997).

${ }^{33}$ S. S. Dhesi, G. van der Laan, H. A. Dürr, M. Belakhovsky, S. Marchesini, P. Kamp, A. Marty, B. Gilles, and A. Rogalev, J. Magn. Magn. Mater. 226-230, 1580 (2001).

${ }^{34}$ M. Finazzi, L. Braicovich, Ch. Roth, F. U. Hillebrecht, H. B. Rose, and E. Kisker, Phys. Rev. B 50, 14671 (1994).

${ }^{35}$ D. Kaptás, J. Balogh, T. Kemény, L. F. Kiss, L. Bujdosó, A. Kovács, A. Hirata, and I. Vincze, Phys. Rev. B 75, 014417 (2007). 